\title{
Effective XPD and XPI Optimization in Reflectarrays for Satellite Missions
}

\author{
Daniel R. Prado, Manuel Arrebola, Senior Member, IEEE
}

\begin{abstract}
Crosspolar requirements for space-borne antennas are very stringent. In some missions such as Direct Broadcast Satellite (DBS) these requirements are specified in terms of crosspolar discrimination (XPD) and crosspolar isolation (XPI), usually with values higher than $33 \mathrm{~dB}$. In this regard, the most common approach for crosspolar optimization for reflectarray antennas is to set up a crosspolar template with certain values, improving the XPD and XPI indirectly. In this work, it is proposed to directly optimize the XPD and XPI in reflectarray antennas with the aim of obtaining better results than the usual crosspolar optimization. For this purpose, the generalized Intersection Approach is employed, for which the forward projection is improved to include XPD and XPI templates. Three optimization strategies are compared: optimizing the crosspolar pattern, the XPD and the XPI. The direct optimization of the XPD and XPI achieves better results in less iterations than the common approach of the crosspolar pattern optimization while keeping the copolar pattern within requirements.
\end{abstract}

Index Terms-Crosspolar discrimination (XPD), crosspolar isolation (XPI), very large reflectarray, Direct Broadcast Satellite (DBS), generalized Intersection Approach

\section{INTRODUCTION}

$\mathbf{R}$ EFLECTARRAYS have been considered for many years as potential substitutes of shaped parabolic reflectors for satellite missions due to their low profile, low cost and easy fabrication [1]. For these applications, very large reflectarrays comprised of thousands of elements are required. Thus, efficient techniques of pattern synthesis are needed that are able to deal with many optimizing variables while obtaining good results. A very efficient algorithm is the Intersection Approach (IA) for Phase-Only Synthesis (POS) [2], which has been used with success for the design of very large reflectarrays for Direct Broadcast Satellite (DBS) applications [3]-[5]. However, the main inconvenience of POS techniques is that only copolar requirements are considered due to some

This work was supported in part by the European Space Agency (ESA) under contract ESTEC/AO/1-7064/12/NL/MH; by the Ministerio de Ciencia, Innovación y Universidades under project TEC2017-86619-R (ARTEINE); by the Ministerio de Economía, Industria y Competitividad under project TEC2016-75103-C2-1-R (MYRADA); by the Gobierno del Principado de Asturias through Programa "Clarín" de Ayudas Postdoctorales / Marie CourieCofund under project ACA17-09.

D. R. Prado is with the Institute of Sensors, Signals and Systems, School of Engineering and Physical Sciences, Heriot-Watt University, Edinburgh, U.K. (email: dr38@hw.ac.uk).

M. Arrebola is with the Department of Electrical Engineering, Group of Signal Theory and Communications, Universidad de Oviedo, Gijón, Spain (e-mail: arrebola@uniovi.es).

Color versions of one or more of the figures in this paper are available online at http://ieeexplore.ieee.org.

Digital Object Identifier XX.XXXX/LAWP.XXXX.XXXXXXX simplifications in the reflectarray analysis. More recently, the crosspolar optimization of reflectarrays has been addressed by performing a direct optimization [6], [7] on the unit cell geometry, since the full unit cell response is necessary to properly characterize the crosspolar pattern. For the crosspolar optimization, the usual approach is to impose some specifications in the crosspolar pattern [6]-[8], although this may not be the optimal approach if the crosspolar pattern is not the designing specification. Such is the case of DBS missions with crosspolar requirements in terms of crosspolar discrimination (XPD) or crosspolar isolation (XPI).

Radiation pattern requirements are usually imposed by means of a set of templates [9], specifying the minimum and maximum requirements. Originally, only the copolar pattern was considered, although it was later extended to include crosspolar requirements in the same manner [7], [8], [10]-[12]. However, since the template is set directly on the crosspolar component of the radiation pattern, the XPD and the XPI are only indirectly optimized [7], obtaining suboptimal results.

In this work, it is proposed to directly optimize the XPD and XPI parameters to improve the cross-polarization performance of reflectarray antennas for space applications, and in particular for DBS. The generalized Intersection Approach (IA) algorithm [13] is employed and improved to directly optimize the XPD and XPI while keeping the copolar pattern within specifications. To that end, new templates based on the XPD and XPI requirements are employed. Three different optimizations are compared: the usual crosspolar optimization, XPD optimization and XPI optimization. Following the proposed new approach, the antenna performance is greatly improved in terms of cross-polarization, while the algorithm performance improves by obtaining better results in less iterations. Although the results are presented only at central frequency, it is expected the same behaviour over a bandwidth when several frequencies are optimized at the same time.

\section{XPD AND XPI OPTIMIZATION WiTH THE GENERALIZED INTERSECTION APPROACH}

The generalized IA is an iterative algorithm which performs two operations at each iteration: the forward and the backward projections [13]. The forward projection computes the current radiation pattern starting from the optimizing variables and then applies the specification templates. On the other hand, the backward projection minimizes the distance between the current radiation pattern and the radiation pattern that fulfills the specifications. In this regard, we start with the implementation of the projectors detailed in [7] and improve the forward 
projection definition to include XPD and XPI templates, apart from the copolar template, necessary to keep the pattern within specifications.

The crosspolar discrimination (XPD) is defined for the coverage area as the ratio (in linear scale) point by point of the copolar and crosspolar gains:

$$
\mathrm{XPD}(u, v)=G_{\mathrm{cp}}(u, v) / G_{\mathrm{xp}}(u, v), \quad \forall(u, v) \in \Omega,
$$

were $u=\sin \theta \cos \varphi, v=\sin \theta \sin \varphi$ and $\Omega$ is a subset of the visible region $\left(u^{2}+v^{2}<1\right)$ corresponding to the coverage area. Usually, the worst XPD value is considered, which corresponds to its minimum:

$$
\mathrm{XPD}_{\min }=\min \operatorname{XDP}(u, v) .
$$

On the other hand, the crosspolar isolation is defined for the coverage area as the ratio (in linear scale) between the minimum copolar gain and the maximum crosspolar gain:

$$
\mathrm{XPI}=G_{\mathrm{cp}, \min }(u, v) / G_{\mathrm{xp}, \max }(u, v), \quad(u, v) \in \Omega .
$$

The goal of the optimization is to increase the XPD and XPI values, so only minimum requirement templates are necessary. Considering $T_{\mathrm{XPD}, \min }(u, v)$ and $T_{\mathrm{XPI}, \min }$ the minimum templates, the following condition should be met:

$$
\begin{aligned}
T_{\mathrm{XPD}, \min }(u, v) & \leq \mathrm{XPD}(u, v), \\
T_{\mathrm{XPI}, \min } & \leq \mathrm{XPI} .
\end{aligned}
$$

Then, in the forward projector of [7] the crosspolar conditions are substituted with (4), taking into account the definitions of the XPD in (1), the minimum XPD in (2) or the XPI in (3). The conditions on the copolar template are maintained. As it will be shown later, it may be more interesting to optimize $\mathrm{XPD}_{\min }$ than the XPD for all points in the coverage zone. In such case, the condition (4) is applied to a single number, as is also the case of the XPI.

\section{RESULTS}

For the examples, all computations are performed in a workstation with two Intel Xeon E5-2650v3, each with ten cores that handle a total of 40 threads at $2.3 \mathrm{GHz}$, and $256 \mathrm{~GB}$ of memory. The chosen algorithm is the generalized IA presented in [7], which has been improved by including templates for the XPD, XPD $\min$ and XPI.

\section{A. Antenna Specifications and Starting Point}

The considered antenna is a large rectangular reflectarray for space applications in single-offset configuration as illustrated in Fig. 1 and comprised of $74 \times 70$ elements in a regular grid. The feed is modeled with a $\cos ^{q} \theta$ function, with $q=23$ and imposing an illumination taper of $-17.9 \mathrm{~dB}$. The feed is placed at coordinates $\vec{r}_{f}=(358,0,1070) \mathrm{mm}$ with regard to the reflectarray center. The periodicity of the unit cell is $14 \mathrm{~mm} \times 14 \mathrm{~mm}$ and the working frequency is $11.85 \mathrm{GHz}$. The antenna is placed in a satellite in geostationary orbit at $10^{\circ}$ longitude.

For the XPD and XPI optimization, the European coverage shown in Fig. 2 is considered in dual-linear polarization and with a minimum copolar requirement of $28 \mathrm{dBi}$ in the

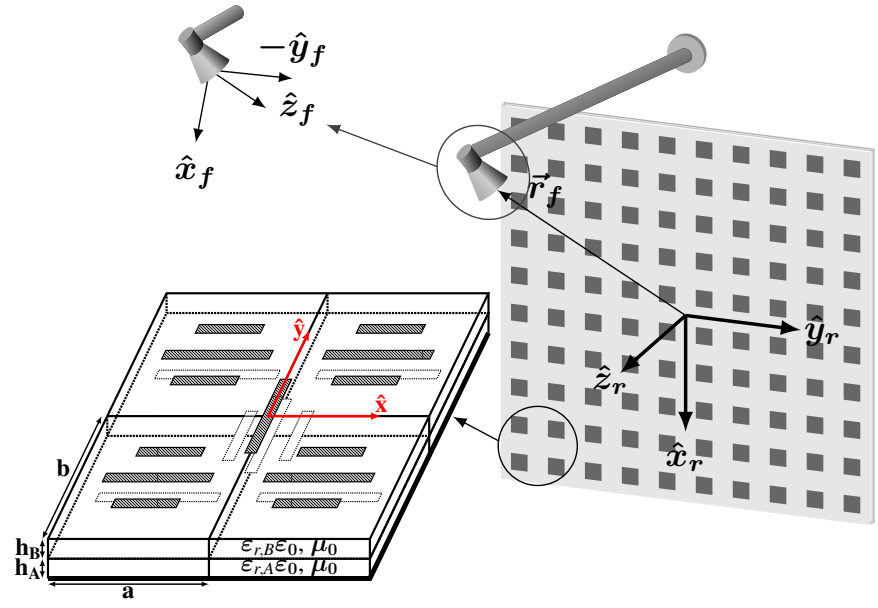

Fig. 1. Sketch of a reflectarray single-offset configuration and the employed unit cell based on two sets of parallel dipoles for dual-linear polarization.

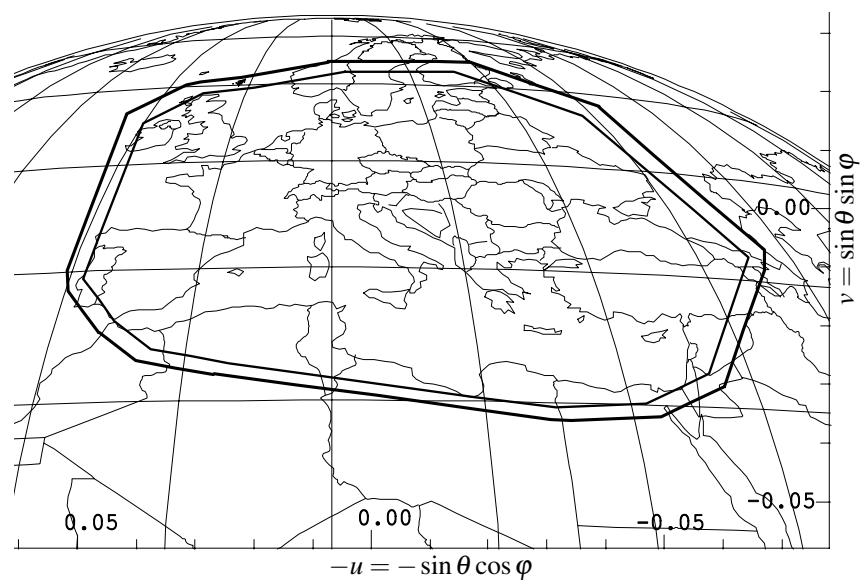

Fig. 2. Europe coverage for DBS application with $(\mathrm{u}, \mathrm{v})$ coordinates in the antenna coordinate system.

coverage. Since the generalized IA is a local optimizer, a proper starting point is necessary. To that end, a two-step approach will be followed. First, a POS to obtain a suitable copolar pattern employing the algorithm detailed in [14] is performed. In this first step, only the phase-shift introduced by the reflectarray elements is optimized, fixing the rest of the parameters, including the feed. Two phase distributions on the reflectarray aperture are obtained which generate the corresponding copolar pattern for each linear polarization. Then, a design is carried out to obtain a reflectarray layout.

The same unit cell as in [7] is used and it is shown in Fig. 1, which is comprised of two sets of four parallel and coplanar dipoles in two layers of metallization. Each set of four dipoles control the phase-shift of a linear polarization. The length of the dipoles are scaled as in [15, Eq. 8], considering one geometrical variable per dipole set (polarization). The substrate for the bottom layer has a height of $h_{A}=2.363 \mathrm{~mm}$ and a complex relative permittivity $\epsilon_{r, A}=2.55-j 2.295 \cdot 10^{-3}$, while the top layer has a height of $h_{B}=1.524 \mathrm{~mm}$ and a complex relative permittivity $\epsilon_{r, B}=2.17-j 1.953 \cdot 10^{-3}$.

Finally, the optimization will be carried out in a subset of 
the whole visible region, corresponding to $u \in(0.05,0.45)$ and $v \in(-0.20,0.15)$ with a resolution of 11187 points in a regular U-V grid. The optimization considers two variables per reflectarray element. Thus, the total number of optimizing variables will be 10360 .

\section{B. Crosspolar, XPD and XPI Optimizations}

In this section, three different approaches will be described to improve the XPD and XPI in the coverage zone. The goal is to demonstrate that directly optimizing the figure of merit (XPD or XPI) provides better results than the usual approach, in which it is optimized indirectly. In all cases, the optimizations are carried out in dual-linear polarization, with all magnitudes in linear scale, and also considering the copolar templates, imposing a minimum value of $28 \mathrm{dBi}$ in the coverage zone in order to maintain the copolar pattern within specifications while the cross-polarization performance is improved.

The first approach (opt. \#1) consists in the optimization of the crosspolar pattern. To this aim, a crosspolar template $40 \mathrm{~dB}$ below the maximum gain is employed in the forward projector of the algorithm, as it is done in [7]. This is the usual approach and it optimizes the XPD and XPI only indirectly.

The second approach (opt. \#2) consists in the XPD optimization. However, after several trials, optimizing the XPD in the whole coverage zone did not provide good results. Indeed, the algorithm minimizes the total error (also considering the copolar template), and tries to improve the XPD in the whole coverage zone. As a result, a critical parameter such as the minimum XPD may worsen despite the fact that the total error decreases. Although a weight function could be used to control the XPD optimization, it is easier to directly optimize $\mathrm{XPD}_{\min }$, with a minimum template of $40 \mathrm{~dB}$ (i.e. the algorithm will try to achieve a $X P D_{\min }$ value of $40 \mathrm{~dB}$ in the coverage area). The template is employed in the forward projector using (4).

Finally, the third optimization (opt. \#3) will optimize the XPI. The XPI is a stricter parameter, and due to its definition, it will be equal or smaller than $\mathrm{XPD}_{\min }$. Thus, by improving $\mathrm{XPI}, \mathrm{XPD}_{\min }$ will also improve. However, the opposite is not true. The selection between XPD and XPI will depend on the mission and its requirements. As in the previous case, the minimum template is set to $40 \mathrm{~dB}$ in the forward projector according to (4).

\section{Results of the Optimizations}

The generalized IA was left to run 60 iterations until the $\mathrm{XPD}_{\min }$ and XPI improvement stagnated in the three cases under study. A summary of the results are shown in Table I. While for the crosspolar optimization both parameters reach a maximum value around $35 \mathrm{~dB}$, values better than $39.5 \mathrm{~dB}$ are achieved with the new technique, which supposes an additional improvement of at least $4.5 \mathrm{~dB}$ over the crosspolar optimization and more than $8 \mathrm{~dB}$ over the starting point for polarization $Y$, and close to a $9 \mathrm{~dB}$ improvement in polarization $X$. In addition, Fig. 3 shows the evolution of $\mathrm{XPD}_{\min }$ and XPI for both linear polarizations and the three optimizations. As it can be seen, the proposed approach to directly optimize the XPD
Table I

RESULTS OF THE DIFFERENT OPTIMIZATION STRATEGIES TO IMPROVE XPD AND XPI AFTER 60 ITERATIONS OF THE GENERALIZED IA.

\begin{tabular}{cccccc}
\hline & \multicolumn{2}{c}{ Polarization X } & & \multicolumn{2}{c}{ Polarization Y } \\
\cline { 2 - 3 } \cline { 5 - 6 } \cline { 5 - 6 } & XPD $_{\min }(\mathrm{dB})$ & $\mathrm{XPI}(\mathrm{dB})$ & & $\mathrm{XPD}_{\min }(\mathrm{dB})$ & $\mathrm{XPI}(\mathrm{dB})$ \\
\hline Initial & 30.85 & 30.63 & & 31.36 & 31.12 \\
Opt. \#1 & 34.99 & 34.74 & & 35.11 & 34.96 \\
Opt. \#2 & 39.63 & 37.45 & & 39.63 & 38.40 \\
Opt. \#3 & 39.66 & 39.54 & & 39.57 & 39.56 \\
\hline
\end{tabular}

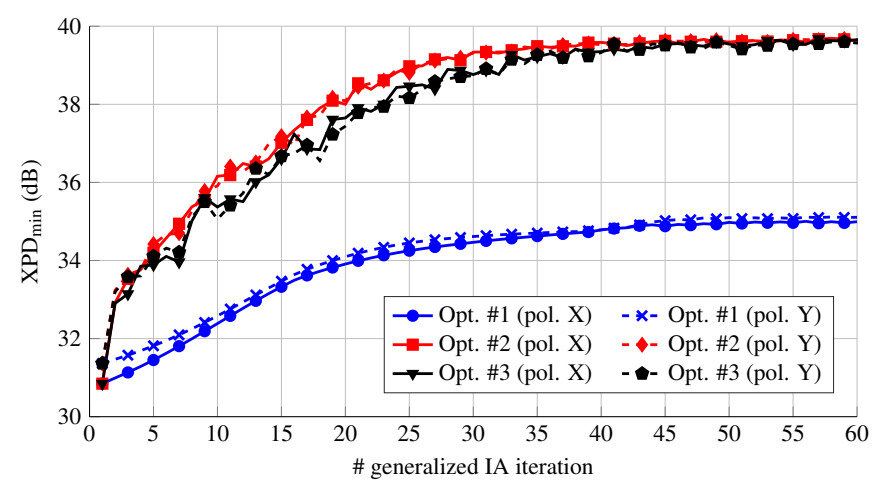

(a)

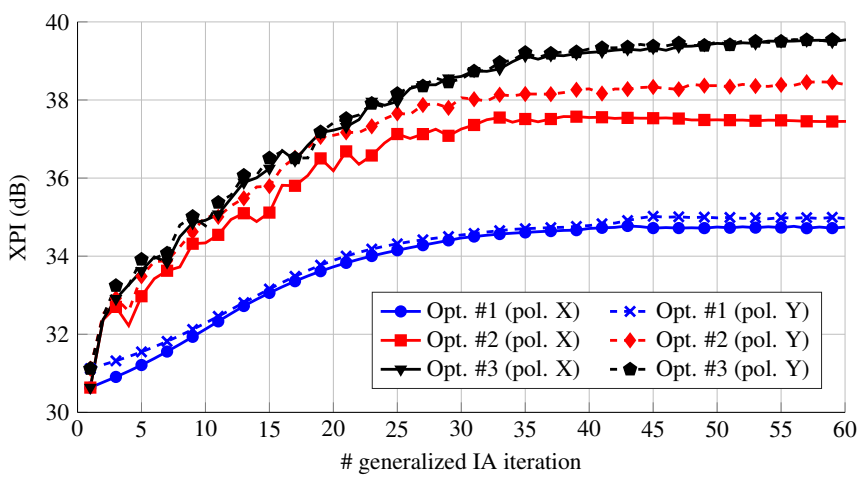

(b)

Fig. 3. Evolution of (a) $\mathrm{XPD}_{\min }$ and (b) XPI for the three different optimization strategies and both linear polarizations.

and XPI offers better results in less iterations. Furthermore, it is observed that when optimizing $\mathrm{XPD}_{\min }$ and $\mathrm{XPI}$, the final value of the $\mathrm{XPD}_{\text {min }}$ is very similar in both methods. However, since the XPI is a stricter parameter, better results are obtained if it is directly optimized instead of $\mathrm{XPD}_{\min }$.

Fig. 4 shows the final copolar and crosspolar patterns for polarization $X$ when the XPI is optimized. The copolar pattern is kept within requirements, having a minimum copolar gain of $28.80 \mathrm{~dB}$. Similar results were obtained for polarization $Y$, for which the minimum copolar gain in the coverage zone is $28.86 \mathrm{~dB}$. Fig. 5 shows the XPD for polarization $X$ at the starting point and the final result after each of the three optimization strategies. The difference between directly optimizing the $\mathrm{XPD}_{\min }$ or the XPI and the crosspolar pattern is mainly noticeable in the edge of the coverage zone. In addition, a more uniform XPD level is obtained along the entire coverage area. 


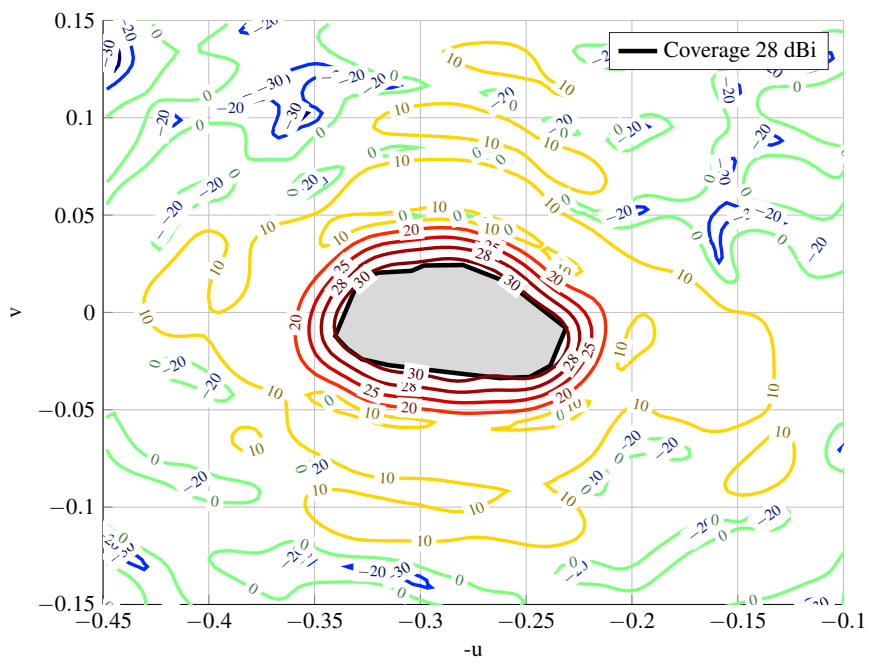

(a)

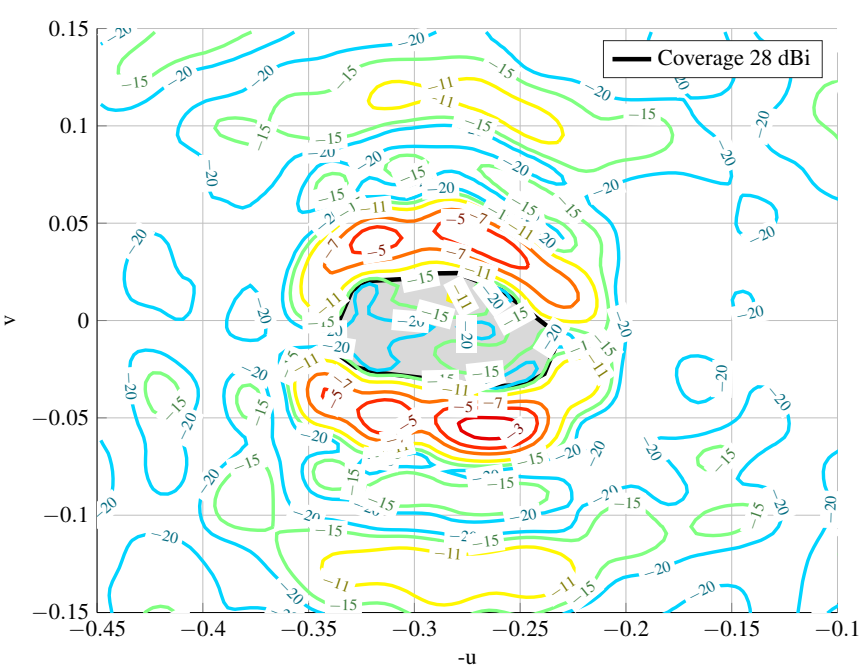

(b)

Fig. 4. Optimized radiation patterns for polarization $X$ with strategy \#3 (XPI optimization). (a) Copolar. (b) Crosspolar.

Finally, Fig. 6 shows the initial and final XPD in the coverage area for both polarizations when the XPI is optimized. For all cases, the losses are estimated to be around $0.12 \mathrm{~dB}$, employing the unit cell and substrate described in Section III.A and the MoM-LP of [16].

Finally, the optimization of $\mathrm{XPD}_{\min }$ or XPI instead of the crosspolar pattern reduces the memory footprint of the optimization algorithm approximately by half.

\section{CONCLUSIONS}

In this letter, the direct optimization of the XPD and XPI is proposed to improve the cross-polarization performace of reflectarray antennas for space applications. In this regard, the generalized IA is employed, improving the definition of the forward projection to include templates for the direct optimization of the XPD and XPI parameters, which are the figures of merit in space applications such as DBS. In this way, better results are obtained in less iterations with regard to the usual approach of imposing a template on the crosspolar

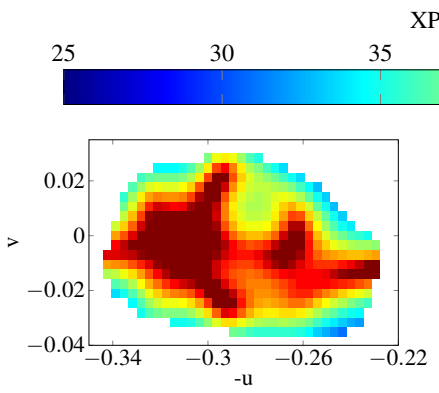

(a)

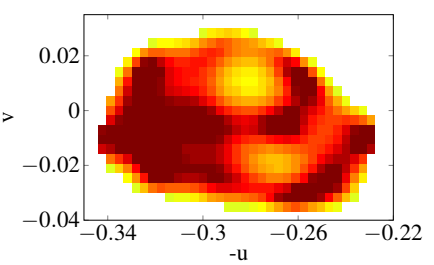

(c)

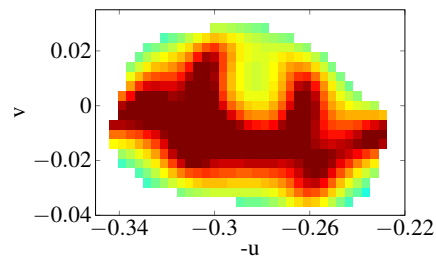

(b)

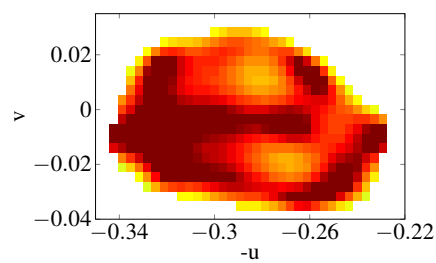

(d)
Fig. 5. Comparison of the XPD obtained for each optimization strategy for polarization $X$. (a) Starting point $\left(\mathrm{XPD}_{\min }=30.85 \mathrm{~dB}\right)$. (b) XP optimization (opt. \#1) $\left(\mathrm{XPD}_{\min }=34.99 \mathrm{~dB}\right)$. (c) $\mathrm{XPD}_{\min }$ optimization (opt. \#2) $\left(\mathrm{XPD}_{\min }\right.$ $=39.63 \mathrm{~dB})$. (d) XPI optimization (opt. \#3) $\left(\mathrm{XPD}_{\min }=39.66 \mathrm{~dB}\right)$.

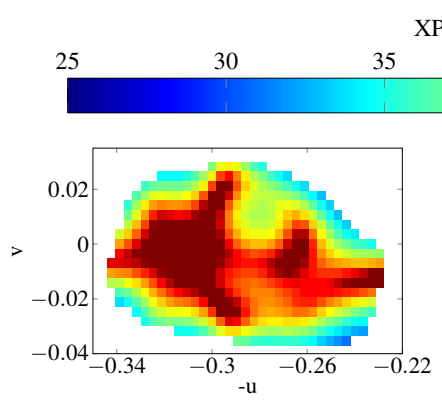

(a)

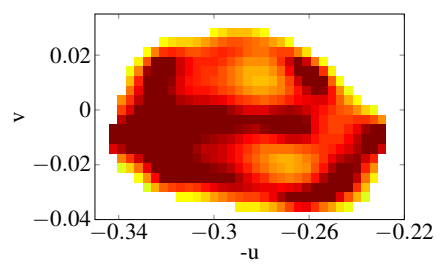

(c)

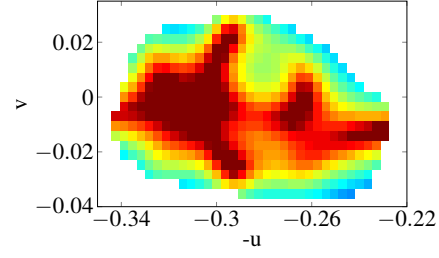

(b)

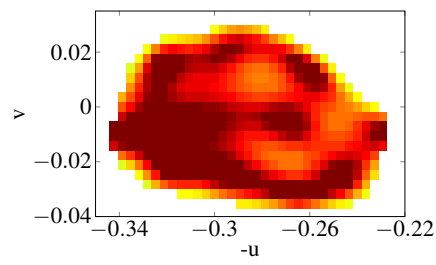

(d)
Fig. 6. XPD before and after the XPI optimization for the European coverage shaped beam. (a) Polarization $X$ before $\left(\mathrm{XPD}_{\min }=30.85 \mathrm{~dB}\right)$. (b) Polarization $Y$ before $\left(\mathrm{XPD}_{\min }=31.36 \mathrm{~dB}\right)$. (c) Polarization $X$ after $\left(\mathrm{XPD}_{\min }=39.66 \mathrm{~dB}\right)$. (d) Polarization $Y$ after $\left(\mathrm{XPD}_{\min }=39.57 \mathrm{~dB}\right)$.

component of the radiation pattern, which only optimizes the XPD and XPI indirectly. Following this approach, an improvement of $4.5 \mathrm{~dB}$ is obtained in the $\mathrm{XPD}_{\text {min }}$ and XPI with regard to the crosspolar optimization, and more than $8 \mathrm{~dB}$ and close to $9 \mathrm{~dB}$ with regard to the starting point for polarizations $Y$ and $X$, respectively.

\section{REFERENCES}

[1] J. Huang and J. A. Encinar, Reflectarray Antennas. Hoboken, NJ, USA: John Wiley \& Sons, 2008.

[2] J. A. Zornoza and J. A. Encinar, "Efficient phase-only synthesis of contoured-beam patterns for very large reflectarrays," Int. J. RF Microw. Comput. Eng., vol. 14, no. 5, pp. 415-423, Sep. 2004. 
[3] J. A. Encinar, L. S. Datashvili, J. A. Zornoza, M. Arrebola, M. SierraCastaner, J. L. Besada-Sanmartin, H. Baier, and H. Legay, "Dualpolarization dual-coverage reflectarray for space applications," IEEE Trans. Antennas Propag., vol. 54, no. 10, pp. 2827-2837, Oct. 2006.

[4] J. A. Encinar, M. Arrebola, L. F. de la Fuente, and G. Toso, "A transmitreceive reflectarray antenna for direct broadcast satellite applications," IEEE Trans. Antennas Propag., vol. 59, no. 9, pp. 3255-3264, Sep. 2011.

[5] J. A. Encinar, R. Florencio, M. Arrebola, M. A. Salas-Natera, M. Barba, J. E. Page, R. R. Boix, and G. Toso, "Dual-polarization reflectarray in Ku-band based on two layers of dipole arrays for a transmit-receive satellite antenna with South American coverage," Int. J. Microw. Wirel. Technol., vol. 10, no. 2, pp. 149-159, 2018.

[6] M. Zhou, S. B. Sørensen, O. S. Kim, E. Jørgensen, P. Meincke, and O. Breinbjerg, "Direct optimization of printed reflectarrays for contoured beam satellite antenna applications," IEEE Trans. Antennas Propag., vol. 61, no. 4, pp. 1995-2004, Apr. 2013.

[7] D. R. Prado, M. Arrebola, M. R. Pino, R. Florencio, R. R. Boix, J. A. Encinar, and F. Las-Heras, "Efficient crosspolar optimization of shaped-beam dual-polarized reflectarrays using full-wave analysis for the antenna element characterization," IEEE Trans. Antennas Propag., vol. 65 , no. 2, pp. 623-635, Feb. 2017.

[8] D. R. Prado, M. Arrebola, M. R. Pino, and F. Las-Heras, "Complex reflection coefficient synthesis applied to dual-polarized reflectarrays with cross-polar requirements," IEEE Trans. Antennas Propag., vol. 63, no. 9, pp. 3897-3907, Sep. 2015.

[9] O. M. Bucci, G. Franceschetti, G. Mazzarella, and G. Panariello, "Intersection approach to array pattern synthesis," IEE Proc. Microw. Antennas Propag., vol. 137, no. 6, pp. 349-357, Dec. 1990.

[10] O. M. Bucci, G. D'Elia, and G. Romito, "Power synthesis of conformal arrays by a generalised projection method," IEE Proc. Microw. Antennas Propag., vol. 142, no. 6, pp. 467-471, Dec. 1995.

[11] O. M. Bucci, A. Capozzoli, G. D'Elia, and S. Musto, "A new approach to the power pattern synthesis of reflectarrays," in Proc. URSI International Symposium on Electromagnetic Theory (EMTS'04), Pisa, Italy, May 2327, 2004, pp. 1053-1055.

[12] A. Capozzoli and G. D'Elia, "Global optimization and antenna synthesis and diagnosis, part two: applications to advanced reflector antennas synthesis and diagnosis techniques," Progr. Electromagn. Res., vol. 56, pp. 233-261, 2006.

[13] O. M. Bucci, G. D'Elia, G. Mazzarella, and G. Panariello, "Antenna pattern synthesis: a new general approach," Proc. IEEE, vol. 82, no. 3, pp. 358-371, Mar. 1994.

[14] D. R. Prado, M. Arrebola, M. R. Pino, and F. Las-Heras, "Improved reflectarray phase-only synthesis using the generalized intersection approach with dielectric frame and first principle of equivalence," Int. J. Antennas Propag., vol. 2017, pp. 1-11, May 2017.

[15] D. R. Prado, J. A. López-Fernández, G. Barquero, M. Arrebola, and F. Las-Heras, "Fast and accurate modeling of dual-polarized reflectarray unit cells using support vector machines," IEEE Trans. Antennas Propag., vol. 66, no. 3, pp. 1258-1270, Mar. 2018.

[16] R. Florencio, R. R. Boix, and J. A. Encinar, "Enhanced MoM analysis of the scattering by periodic strip gratings in multilayered substrates," IEEE Trans. Antennas Propag., vol. 61, no. 10, pp. 5088-5099, Oct. 2013. 